\title{
Rational ergodicity of geodesic flows
}

\author{
JON AARONSON AND DENNIS SULLIVAN \\ Department of Mathematics, Tel Aviv University, Tel Aviv, Israel; IHES, 35 Route \\ de Chartres, 91440 Bures-sur-Yvette, France
}

(Received 14 March 1983 and revised 11 July 1983)

Abstract. We prove the rational egodicity of geodesic flows on divergence type surfaces of constant negative curvature, and identify their asymptotic types.

\section{Introduction}

We discuss recurrence and transitivity properties of geodesics on hyperbolic surfaces (complete, two dimensional Riemannian manifolds with constant curvature -1 ). Every such surface has the unit disc as universal cover and can be viewed as $H / \Gamma$ where $H$ is the unit disc equipped with the hyperbolic metric and $\Gamma$ is the covering group of isometries of $H$. If $\mathscr{F}$ is a fundamental domain for $\Gamma$ in $H$ then $H / \Gamma$ can be pictured as $\mathscr{F}$ with some identifications on $\partial \mathscr{F}$.

Let $N$ be a small ball in the hyperbolic surface $H / \Gamma, l$ be a directed geodesic on $H / \Gamma$ and $x \in l$. Let $x(l, t)$ denote the point on $l$ which is directed distance $t$ from $x$. We discuss the property,

$$
\int_{0}^{\infty} 1_{N}(x(l, t)) d t=\infty
$$

for almost all geodesics $l$ and in this case, we try to say something about the rate at which $\int_{0}^{T} 1_{N}(x(l, t)) d t$ tends to infinity, as $T$ tends to infinity.

This property is better viewed in terms of the geodesic flow of the surface which is defined on the space of line elements on the surface (the set of points equipped with directions). The space of line elements on the surface $H / \Gamma$ may be viewed as $X_{\Gamma}=\mathscr{F} \times[0,2 \pi)$ and the geodesic flow, $\varphi_{\Gamma}^{t}: X_{\Gamma} \rightarrow X_{\Gamma}$ preserves the measure $m_{\Gamma}=$ Hyperbolic area $\times$ Lebesgue measure.

The above mentioned property now boils down to

$$
\int_{0}^{\infty} 1_{\Delta} \cdot \varphi_{\Gamma}^{t}(\omega) d t=\infty \quad \text { for } m_{\Gamma} \text {-a.e. } \omega \in X_{\Gamma} \text {, where } \Delta=N \times[0,2 \pi) \text {. }
$$

In the case where the surface $H / \Gamma$ has finite area (and $m_{\Gamma}\left(X_{\Gamma}\right)<\infty$ ), E. Hopf [6] proved that the geodesic flow is ergodic which implies that $\int_{0}^{\infty} 1_{\Delta} \cdot \varphi_{\Gamma}^{t} d t=\infty$ $m_{\Gamma}$-a.e. for every $N$, and indeed, by Birkhoff's ergodic theorem, that

$$
\int_{0}^{T} 1_{\Delta} \cdot \varphi_{\Gamma}^{t} d t / T \underset{T \rightarrow \infty}{\longrightarrow} m_{\Gamma}(\Delta) / m_{\Gamma}\left(X_{\Gamma}\right) \quad m_{\Gamma} \text {-a.e. }
$$

In 1939, Hopf, using his ratio ergodic theorem [7], proved that geodesic flows on hyperbolic surfaces of infinite area are either totally dissipative $\left(\int_{0}^{\infty} f \cdot \varphi_{\Gamma}^{t} d t<\infty\right.$ 
a.e. for every $\left.f \in L_{+}^{1}\left(m_{\Gamma}\right)\right)$ or conservative and ergodic $\left(\int_{0}^{\infty} f \cdot \varphi_{\Gamma}^{t} d t=\infty\right.$ for every $\left.f \in L_{+}^{1}\left(m_{\Gamma}\right), \int f>0\right)$.

The 1939 result of Hopf is the only one about geodesic flows which we assume. (A more accessible proof is given in [9]). These results extend canonically to geodesic flows of arbitrary dimension.

If the integral $\int_{0}^{\infty} m_{\Gamma}\left(\Delta \cap \varphi_{\Gamma}^{-t} \Delta\right) d t$ converges, it is immediate that the geodesic flow $\varphi_{\Gamma}$ cannot be conservative. It is more difficult to show that if this integral diverges, then the geodesic flow is not totally dissipative. This was done by M. Tsuji [18], using complex function theory. We shall give a simpler proof of this result (which generalises easily to arbitrary dimension) by proving (lemma 1) that:

$$
\sup \int_{\Delta} S_{t}\left(1_{\Delta}\right)^{2} d m_{\Gamma} /\left(\int_{\Delta} S_{t}\left(1_{\Delta}\right) d m_{\Gamma}\right)^{2}<\infty
$$

for $\Delta=N \times(0,2 \pi)$ where $S_{t}(f)=\int_{0}^{t} f \cdot \varphi_{\Gamma}^{s} d s$, and then using a continuous time version (lemma 2) of the Borel-Cantelli lemma of Renyi [14, p. 391]) to show that if $\int_{0}^{\infty} m_{\Gamma}\left(\Delta \cap \varphi_{\Gamma}^{-t} \Delta\right) d t=\infty$ then $\varphi_{\Gamma}$ is not totally dissipative.

Lemma 1 is proved by inspecting $m_{\Gamma}\left(\Delta \cap \varphi_{\Gamma}^{-t} \Delta\right)$ in terms of the arrangement of the lifts of $N$ in $H$. The quantity $m_{\Gamma}\left(\Delta \cap \varphi_{\Gamma}^{-1} \Delta\right)$ is closely related to the angle subtended at points of $N$ (lifted) by translates $\gamma N$ of $N$ at a distance approximately $t$ from $N$, and indeed the integral $\int_{0}^{\infty} m_{\Gamma}\left(\Delta \cap \varphi_{\Gamma}^{-t} \Delta\right) d t$ diverges iff the total angle subtended by all translates $\gamma N$ of $N$ at points of $N$ is infinite. The angle subtended by $\gamma N$ at points of $N$ is approximately 4 diam $N e^{-2 \rho_{\gamma}}$ where $\rho_{\gamma}$ is the hyperbolic distance between the centres of $N$ and $\gamma N$. In the case where $N$ is centred at 0 then $4 e^{-2 \rho_{\gamma}} \sim 1-|\gamma(0)|^{2}$. The series $\sum_{\gamma \in \Gamma}(1-|\gamma(0)|)^{s}, s>0$, is known as the Poincare series of $\Gamma$ (see proposition 1 ). We have outlined a new proof of:

THEOREM 1. The geodesic flow on the hyperbolic surface $H / \Gamma$ is conservative and ergodic if and only if the Poincaré series of $\Gamma$ diverges at $s=1$.

Such surfaces are called of divergence type.

The main point of this paper (besides the elementary proof of theorem 1 - see also [17, chap. 8]) is to say something about the growth rate to infinity of

$$
S_{t}\left(1_{\Delta}\right)=\int_{0}^{t} 1_{\Delta} \cdot \varphi_{\Gamma}^{s} d s
$$

for $\varphi_{\Gamma}$ an ergodic geodesic flow on a hyperbolic surface of infinite area. We do this by showing that an ergodic geodesic flow of a hyperbolic surface is rationally ergodic and identifying its asymptotic type (theorem 2). We now proceed to explain these terms and their relevance to growth rates.

Let $\left(X, \mathscr{B}, \mu, T^{t}\right)$ be a conservative ( = recurrent) ergodic measure preserving flow of a $\sigma$-finite measure space. Set

$$
S_{\imath}(f)=\int_{0}^{t} f \cdot T^{s} d s \quad \text { for } f \in L^{1}(\mu) .
$$

Then, for $f \in L_{+}^{1}, S_{t}(f) \uparrow \infty$ a.e. and one may ask for its asymptotic growth rate as $t \rightarrow \infty$. 
If $\mu(X)=1$ then the Birkhoff ergodic theorem states that $S_{t}(f)(x) / t \rightarrow_{t \rightarrow \infty} \int_{X} f d \mu$ for $\mu$-a.e. $x, f \in L^{1}$, and so the growth rate of $S_{t}(f)(x)\left(f \in L_{+}^{1}\right)$ is independent of $f \in L_{+}^{1}$ and $x \in X(\bmod \mu)$.

If $\mu(X)=\infty$ then the Hopf ratio ergodic theorem states that $S_{t}(f)(x) / S_{t}(g)(x) \rightarrow$ $\int_{X} f d \mu / \int_{X} g d \mu$ for $\mu$-a.e. $x \in X, f, g \in L^{1}, \int g \neq 0$. This shows that the growth rate of $S_{t}(f)(x)$ is independent of $f \in L_{+}^{1}$, but the only 'absolute' information (w.r.t. $x \in X$ ) that can be deduced is that

$$
S_{t}(f)(x) / t \rightarrow 0 \quad \text { for } \mu \text {-a.e. } x \in X, f \in L_{+}^{1} \text {. }
$$

A search for 'absolute' growth rates for $S_{t}(f)(x)$ (those independent of $f \in L_{+}^{1}$ and $x \in X \bmod \mu$ ) leads to a study of properties like:

$$
S_{t}(f) / a(t) \rightarrow \int_{X} f d \mu \quad \text { in some sense, for all } f \in L^{1},
$$

where $a(t)$ are constants.

Unfortunately, when $\mu(X)=\infty$, there are never constants $a(t)$ which satisfy the convergence (3) in the a.e. sense (see [1] for the analogous result for transformations, which easily implies this). However, there are flows with $\mu(X)=\infty$, for which there are constants which satisfy the convergence (3) in weaker senses. We say $f_{t} \in L^{1}$ converges feebly to $g \in L^{1}$ if every subsequence of $\left\{f_{t}\right\}$ has a subsequence whose Çesàro means converge to $g$ a.e., and write $f_{t} \rightarrow \leadsto g$. For some (but not all) ergodic measure preserving flows on $X,(\mu(X)=\infty)$, there are constants $a(t)$ for which

$$
S_{t}(f) / a(t)-\cdots \int_{X} f d \mu
$$

for some, (and hence all, by Hopf's ratio theorem) $f \in L^{1}$. (The analogous property for transformations is discussed in [3]). For such flows, the $a(t)$ are uniquely determined up to asymptotic equality and their growth rate is called the asymptotic type of the flow. A sufficient condition for (4) is the property rational ergodicity (see [2]).

A measure preserving flow $\left(X, \mathscr{B}, \mu, T^{t}\right)$ is said to be rationally ergodic if there is a set $A \in \mathscr{B}, 0<\mu(A)<\infty$ and a constant $M$ so that

$$
\int_{A} S_{t}\left(1_{A}\right)^{2} d \mu \leq M\left(\int_{A} S_{t}\left(1_{A}\right) d \mu\right)^{2} \quad \text { for } t \geq 1 .
$$

The asymptotic type of $T^{t}$ is then

$$
a(t)=\frac{1}{\mu(A)^{2}} \int_{A} S_{t}\left(1_{A}\right) d \mu .
$$

The inequality (2) is (5) for the geodesic flow. Our main result is:

THEOREM 2. (a) The geodesic flow on a hyperbolic surface of divergence type is rationally ergodic.

(b) The asymptotic type is given by

$$
a(t) \sim \sum_{\substack{\gamma \in \Gamma \\ \rho(x, \gamma(y)) \leq t}} e^{-2 \rho(x, \gamma(y))} \quad \text { any } x, y \in H .
$$


It follows from results of $M$. Rees [13] that for $H / \Gamma$ a $\mathbb{Z}^{\nu}$ cover, $\nu=1,2$, of a compact surface, $a_{\Gamma}(t)$ grows as $t^{\frac{1}{2}},(\nu=1), \log t,(\nu=2)$.

Theorem 1 (in dimension 2) was noted by Nicholls [10] who combined the results of [18] and [8]. Independently the second author proved the theorem (in all dimensions) ([15], [16]) using Brownian motion to get the equivalence of:

(i) ergodicity of the geodesic flow on $X / \Gamma$;

(ii) recurrence of Brownian motion on $H / \Gamma$;

(iii) the non-existence of a positive finite Green's function;

(iv) the divergence of the Poincare series;

where: (ii) $\Leftrightarrow$ (iii) is well known in the theory of Markov processes, (iii) $\Leftrightarrow$ (iv) in dimension 2 is due to Poincaré [12] (see also [18]) and (i) $\Leftrightarrow$ (ii) is based on the fact that a Brownian path in $H$ has an angular limit at $\infty$ almost surely.

Properties (ii) and (iii) make the 'construction' of examples quite easy: let $\Lambda$ be any closed subset of the Riemann sphere $\hat{C}$ containing at least 3 points; then the Riemann surface $\hat{C}-\Lambda=M$ has universal cover $H$ and so admits a hyperbolic metric. Brownian motion on $M$ with the hyperbolic metric is equivalent to a time change to Brownian motion on $\hat{\mathbb{C}}$ stopped at $\Lambda$. Thus, Brownian motion on $M$ is recurrent iff $\Lambda$ is invisible to almost all paths in $\hat{\mathbb{C}}$. This happens precisely when $\Lambda$ has logarithmic capacity zero (for every positive measure $\mu$ on $\Lambda$ :

$$
G(x)=\int_{\Lambda} \log |x-y| d \mu(y) \text { is infinite }
$$

compare (iii)).

One knows that the hyperbolic area of $M$ is infinite as soon as $\Lambda$ is infinite. So we have many examples of infinite volume, hyperbolic surfaces whose geodesic flows are ergodic.

This article is about surfaces in order to preserve simplicity. Analogous theorems hold in arbitrary dimensions. Divergence type in dimension $d+1$ is $\sum_{\gamma \in \Gamma} e^{-2 d \rho(x, \gamma(y))}=\infty$ and the asymptotic type of the geodesic flow on a divergence type manifold is $a(t)=\sum_{\rho(x, y(y)) \leq t} e^{-2 d \rho(x, y(y))}$.

\section{Notation}

We establish notation by recalling the definition of the geodesic flow on a hyperbolic surface, beginning with

$$
H=\left\{z=(u, v) \in \mathbb{R}^{2}:|z|=\sqrt{u^{2}+v^{2}}<1\right\} .
$$

(Readers wishing a more complete recall are referred to [9]). The hyperbolic geometry on $H$ is given by the arc length element

$$
d s(z)=\sqrt{d u^{2}+d v^{2}} /\left(1-|z|^{2}\right)
$$

and the hyperbolic area element

$$
d A(z)=d u d v /\left(1-|z|^{2}\right)^{2}
$$

(here $z=(u, v)$ ). The hyperbolic distance between two points $x, y \in H$ is defined as

$$
\rho(x, y)=\inf \left\{\int_{\gamma} d s: \alpha \text { is an arc joining } x \text { and } y\right\},
$$


and it turns out that $\rho(x, y)=\tanh ^{-1}|(x-y) /(1-\bar{x} y)|$, (here the multiplication is complex). The isometries of $H$ (i.e. transformations of $H$ preserving hyperbolic distance) are precisely the Möbius transformations of $H(z \rightarrow(\lambda z-\alpha) /(1-\bar{\alpha} z)$, where $|\lambda|=1$ and $\alpha \in H$ ) and their complex conjugates ( $z \rightarrow \overline{g(z)}$ where $g$ is Möbius).

The geodesics in $H$ (arcs in $H$ for which the $d s$-length of any segment is the hyperbolic distance between the endpoints of the segment) turn out to be diameters of $H$ and circles orthogonal to $\partial H=\left\{z \in \mathbb{R}^{2}:|z|=1\right\}$. A geodesic meets $\partial H$ at two end-points, and is actually characterized by these two points. A geodesic can be directed by ordering these points, and then the geodesic is considered as directed away from its first endpoint (and towards its second).

The space of line elements of $H$ is $H \times[0,2 \pi)$. To each line element $\omega=$ $(x(\omega), \theta(\omega)) \in X$ there corresponds a unique directed geodesic passing through $x(\omega)$, whose directed tangent at $x(\omega)$ makes an angle $\theta$ with the radius $(0,1) \subseteq H$. The geodesic flow transformation $\varphi^{\prime}$ is defined as follows at $\omega$. If $t>0$, the point $x\left(\varphi^{i} \omega\right)$ is the unique point on the geodesic at hyperbolic distance $t$ from $x(\omega)$ in the direction of the geodesic (away from its first endpoint). If $t<0$ the point $x\left(\varphi^{t} \omega\right)$ is the unique point on the geodesic at hyperbolic distance $-t$ from $x(\omega)$ in the opposite direction of the geodesic (towards its first endpoint). The direction $\theta\left(\varphi^{t} \omega\right)$ is the angle made by the directed tangent of the directed geodesic at the point $x\left(\varphi^{t} \omega\right)$, with the radius $(0,1)$. For example: $\varphi^{t}(0, \theta)=\left(\tanh t e^{i \theta}, \theta\right)$. Isometries $g$ of $H$ also act on $X$ in the natural manner:

$$
\begin{gathered}
g(\omega)=(g(x(\omega)), \text { direction of tangent of } g(\vec{l}) \text { wherever } \vec{l} \text { passes through } \\
x(\omega) \text { in direction } \theta(\omega))
\end{gathered}
$$

It turns out that $\varphi^{t} \circ g=g \circ \varphi^{t}$ wherever $g$ is an isometry of $H$. Both the geodesic flow transformations and isometries preserve the measure $d m(x, \theta)=d A(x) d \theta$ on $X$.

Now let $\Gamma$ be a discrete group of isometries of $H$ (i.e. $\Gamma(x)$ has no cluster point in $H$ for $x \in H)$. The surface $H / \Gamma$ is defined to be $H / \Gamma=\{\Gamma(x): x \in H\}$.

A typical geodesic in $H / \Gamma$ is given by $\Gamma(l)$ where $l$ is a geodesic in $H$. It turns out that the space of line elements in $H / \Gamma$ is given by $X_{\Gamma}=X / \Gamma=\{\Gamma(\omega): \omega \in X\}$ and the geodesic flow on $X_{\Gamma}$ is given by

$$
\varphi_{\Gamma}^{\prime} \Gamma(\omega)=\Gamma\left(\varphi^{t} \omega\right)
$$

The surface $H / \Gamma$ may be pictured intuitively as a fundamental domain for $\Gamma$ in $H$, that is, a set $\mathscr{F} \subseteq H$ with the property that for every $x \in H$ there is unique $\gamma \in \Gamma$ so that $\gamma(x) \in \mathscr{F}$ (e.g. $\bar{F}=\{x \in H: \rho(x, 0) \leq \rho(x, \gamma(0))$ for all $\gamma \neq e, \gamma \in \Gamma\})$ and $X_{\mathrm{r}} \cong \mathscr{F} \times[0,2 \pi)$.

Via this picture, the measures $A$ on $H$, and $m$ on $X$ project naturally onto $A_{\Gamma}$ on $H$ and $m_{\Gamma}$ on $X_{\Gamma}$ respectively.

For geometric constructions of fundamental domains, see [4].

\section{Recurrence of geodesics and the Poincaré series}

In this section, we prove theorems 1 and 2 by studying the amount of time spent by geodesics in small balls of the surface. To fix ideas, let $\mathscr{F}_{\Gamma}$ be a fundamental 
domain for $\Gamma$. Let $x, y \in \mathscr{F}_{\Gamma}^{0}$, and $\varepsilon>0$ be so that

$$
N_{\rho}(x, 3 \varepsilon), N_{\rho}(y, 3 \varepsilon) \subseteq \mathscr{F}_{\Gamma}^{0} .
$$

(Here, $\left.N_{\rho}(x, \varepsilon)=\{z \in H: \rho(x, z)<\varepsilon\}\right)$. Set

$$
\Delta_{x}=N_{\rho}(x, \varepsilon) \times[0,2 \pi) \subseteq \mathscr{F}_{\Gamma} \times[0,2 \pi)
$$

and let $\Delta_{x}^{\Gamma}$ be the projection of $\Delta_{x}$ in $X_{\Gamma}$. We first study the average amount of time spent in $\Delta_{y}^{\Gamma}$ by geodesics starting in $\Delta_{x}^{\Gamma}$. Set, for $t>0$ :

$$
a_{\Gamma}(x, y ; t)=\sum_{\substack{\gamma \in \Gamma \\ \rho(x, \gamma(y)) \leq t}}\left(1-\tanh ^{2} \rho(x, \gamma(y))\right)=a(x, y ; t)
$$

Proposition 1. $\int_{0}^{\infty} m_{\Gamma}\left(\Delta_{x}^{\Gamma} \cap \varphi_{\Gamma}^{-s} \Delta_{y}^{\Gamma}\right) d s=\infty$ iff $a(x, y ; t) \rightarrow_{t \rightarrow \infty} \infty$, and in this case:

$$
\begin{aligned}
& \varliminf_{t \rightarrow \infty} \frac{1}{a(x, y ; t)} \int_{0}^{t} m_{\Gamma}\left(\Delta_{x}^{\Gamma} \cap \varphi_{\Gamma}^{-s} \Delta_{y}^{\Gamma}\right) d s \geq e^{-4 \varepsilon}\left(\frac{m_{\Gamma}\left(\Delta_{x}^{\Gamma}\right)^{2}}{4 \pi^{2}}\right) \\
& \varlimsup_{t \rightarrow \infty} \frac{1}{a(x, y ; t)} \int_{0}^{t} m_{\Gamma}\left(\Delta_{x}^{\Gamma} \cap \varphi_{\Gamma}^{-s} \Delta_{y}^{\Gamma}\right) d s \leq e^{4 \varepsilon}\left(\frac{m_{\Gamma}\left(\Delta_{x}^{\Gamma}\right)^{2}}{4 \pi^{2}}\right)
\end{aligned}
$$

Proof. Set $\alpha(t)=\int_{0}^{t} m_{\Gamma}\left(\Delta_{x}^{\Gamma} \cap \varphi_{\Gamma}^{-s} \Delta_{y}^{\Gamma}\right) d s$. Then

$$
\alpha(t)=\int_{N_{\rho}(x, \varepsilon)} \Phi(t, z) d A(z),
$$

where

$$
\Phi(t, z)=\sum_{\gamma \in \Gamma} \int_{0}^{2 \pi} \int_{0}^{t} 1_{\gamma \Delta_{y}} \cdot \varphi^{s}(z, \theta) d s d \theta .
$$

Setting $\Phi_{z}(w)=z+w / 1+\bar{z} w$, and using $\Phi_{z} \cdot \varphi^{s}=\varphi^{s} \cdot \Phi_{z}$,

$$
\begin{aligned}
\Phi(t, z) & =\sum_{\gamma \in \Gamma} \int_{0}^{2 \pi} \int_{0}^{t} 1_{\Phi_{z}^{-1} \gamma \Delta_{y}} \cdot \varphi^{s}(0, \theta) d s d \theta \\
& =\sum_{\gamma \in \Gamma} \int_{0}^{2 \pi} \int_{0}^{1} 1_{\Phi_{z}^{-1} \gamma N_{\rho}(y, \varepsilon)}\left(\tanh s e^{i \theta}\right) d s d \theta \\
& =\sum_{\gamma \in \Gamma} \int_{0}^{2 \pi} \int_{0}^{\tanh t} 1_{\Phi_{z}^{-1} \gamma N_{\rho}(y, \varepsilon)}\left(r e^{i \theta}\right) \frac{d r d \theta}{1-r^{2}} \\
& =\sum_{\gamma \in \Gamma} \int_{N_{\rho}(0, t)} \frac{1-|w|^{2}}{|w|} \cdot 1_{N_{\rho}\left(\Phi_{z}^{-1} \gamma(y), \varepsilon\right)}(w) d A(w)
\end{aligned}
$$

Now suppose that $w \in N_{\rho}\left(\Phi_{z}^{-1} \gamma(y), \varepsilon\right)$, then

$$
|\rho(\gamma(y), z)-\rho(0, w)|<\varepsilon .
$$

Thus:

$$
\begin{aligned}
\Phi(t, z) & \geq \sum_{\gamma \in \Gamma}\left(1-\tanh ^{2}(\rho(\gamma(y), z)+\varepsilon)\right) A\left(N_{\rho}(0, t) \cap N_{\rho}\left(\Phi_{z}^{-1} \gamma(y), \varepsilon\right)\right) \\
& \geq \sum_{\substack{\gamma \in \Gamma \\
\rho(\gamma(y), z) \leq t-\varepsilon}}\left(1-\tanh ^{2}(\rho(\gamma(y), z)+\varepsilon)\right) \cdot A\left(N_{\rho}(y, \varepsilon)\right)
\end{aligned}
$$


and, since $\rho(z, x)<\varepsilon$ :

$$
\begin{aligned}
\Phi(t, z) & \geq A\left(N_{\rho}(y, \varepsilon)\right) \sum_{\substack{\gamma(y \in \Gamma \\
\rho(y), x) \leq t-2 \varepsilon}}\left(1-\tanh ^{2}(\rho(\gamma(y), x)-2 \varepsilon)\right) \\
& \geq e^{-4 \varepsilon} A\left(N_{\rho}(y, \varepsilon)\right) a(x, y ; t-2 \varepsilon)
\end{aligned}
$$

since

$$
e^{-2 \delta} \leq \frac{1-\tanh ^{2}(t \pm \delta)}{1-\tanh ^{2} t}=\frac{\cosh ^{2} t}{\cosh ^{2}(t \pm \delta)} \leq e^{2 \delta} \quad \text { for } \delta>0
$$

whence

$$
\alpha(t) \geq e^{-4 \varepsilon} A\left(N_{\rho}(0, \varepsilon)\right)^{2} a(x, y ; t-2 \varepsilon)=e^{-4 \varepsilon} \frac{1}{4 \pi^{2}} m_{\Gamma}(\Delta)^{2} a(x, y ; t-2 \varepsilon) .
$$

Also:

$$
\begin{aligned}
\Phi(t, z) & \leq \sum_{\gamma \in \Gamma}\left(1-\tanh ^{2}(\rho(z, \gamma(y))-\varepsilon)\right) \int_{N_{\rho}(0, t)} \frac{1}{|w|} 1_{N_{\rho}\left(\Phi \bar{z}^{-1} \gamma(y), \varepsilon\right)}(w) d A(w) \\
& \leq \sum_{\substack{\gamma \in \Gamma \\
\rho(z, \gamma(y)) \leq t+\varepsilon}}\left(1-\tanh ^{2}(\rho(z, \gamma(y)-\varepsilon)) \int_{N_{\rho}\left(\Phi \bar{z}^{-1} \gamma(y), \varepsilon\right)} \frac{1}{|w|} d A(w) .\right.
\end{aligned}
$$

Now, for $\gamma \neq e$

$$
\begin{aligned}
\int_{N_{\rho}\left(\Phi_{z}^{-1} \gamma(y), \varepsilon\right)} \frac{1}{|w|} d A(w) & \leq \frac{1}{\tanh (\rho(\gamma(y), z)-\varepsilon)} A\left(N_{\rho}(y, \varepsilon)\right) \\
& \leq \frac{1}{\tanh \varepsilon} A\left(N_{\rho}(y, \varepsilon)\right)
\end{aligned}
$$

Hence

$$
\alpha(t) \leq \text { const. } a(x, y ; t+2 \varepsilon) .
$$

This proves that $\alpha(t) \uparrow \infty$ iff $a(x, y ; t) \uparrow \infty$. Noting that $\int_{N_{\rho}\left(\Phi_{z}^{-1} \gamma(y), \varepsilon\right)}(1 /|w|) d A(w) \rightarrow$ $A\left(N_{\rho}(y, \varepsilon)\right)$ as $\rho(x, \gamma(y)) \rightarrow \infty$ uniformly in $z \in N_{\rho}(x, \varepsilon)$, we see that when $\alpha(t) \uparrow \infty$ :

$$
\varlimsup_{t \rightarrow \infty} \frac{\alpha(t)}{a(x, y, t+2 \varepsilon)} \leq e^{4 \varepsilon} A\left(N_{\rho}(0, \varepsilon)\right)^{2}=e^{4 \varepsilon}\left(\frac{1}{4 \pi^{2}} m_{\Gamma}(\Delta)^{2}\right) \text {. }
$$

Now, when $\alpha(t) \uparrow \infty$, necessarily $\alpha(t+\varepsilon) \sim \alpha(t)$ as $t \rightarrow \infty$ for all $\varepsilon>0,\left(0 \leq \alpha^{\prime}(t) \leq\right.$ $m_{\Gamma}(\Delta)$, so (2.3) and (2.4) now yield (2.1) and (2.2).

Next, we prove slightly more than the inequality (5) for the sets $\Delta_{x}^{\Gamma}$.

Lemma 1. For every $x, y \in \mathscr{F}_{\Gamma}$ and $\varepsilon>0$ so that $N_{\rho}(x, 3 \varepsilon), N_{\rho}(y, 3 \varepsilon) \subseteq \mathscr{F}_{\Gamma}$ there is $a$ constant $M<\infty$ so that

$$
\int_{\Delta_{y}^{\Gamma}} S_{t}\left(1_{\Delta_{x}^{\Gamma}}\right)^{2} d m_{\Gamma} \leq M\left(\int_{\Delta_{y}^{\Gamma}} S_{t}\left(1_{\Delta_{x}^{\Gamma}}\right) d m_{\Gamma}\right)^{2}
$$

where

$$
S_{t}\left(1_{\Delta_{x}^{\Gamma}}\right)=\int_{0}^{t} 1_{\Delta_{x}^{\Gamma}} \cdot \varphi_{\Gamma}^{s} d s
$$


Proof. Writing $\Delta_{x}=N_{\rho}(x, \varepsilon) \times[0,2 \pi)$, and $\Delta_{x}^{\Gamma}$ for the projection of $\Delta_{x}$ in $X_{\Gamma}$

$$
\begin{aligned}
\int_{\Delta_{y}^{\Gamma}} S_{t}\left(1_{\Delta_{x}}\right)^{2} d m_{\Gamma} & =2 \int_{0}^{t} \int_{u}^{t} m_{\Gamma}\left(\Delta_{y}^{\Gamma} \cap \varphi_{\Gamma}^{-u} \Delta_{x}^{\Gamma} \cap \varphi_{\Gamma}^{-v} \Delta_{x}^{\Gamma}\right) d u d v \\
& =2 \int_{N_{p}(y, e)} \psi(t, z) d A(z),
\end{aligned}
$$

where

$$
\begin{aligned}
\psi(t, z) & =\sum_{\beta, \gamma \in \Gamma} \int_{0}^{2 \pi} \int_{0}^{t} \int_{u}^{t} 1_{\beta \Delta_{x}} \cdot \varphi^{u}(z, \theta) 1_{\gamma \Delta_{x}} \cdot \varphi^{v}(z, \theta) d u d v d \theta \\
& =\int_{0}^{t} \sum_{\beta \in \Gamma_{u}} \int_{0}^{2 \pi} 1_{\Phi_{z}^{-1} \beta N}\left(\tanh u e^{i \theta}\right) \sum_{\gamma \in \Gamma(\beta)} \int_{u}^{t} 1_{\Phi_{z}^{-1} \gamma N}\left(\tanh v e^{i \theta}\right) d v d \theta d u,
\end{aligned}
$$

where $\Gamma_{u}=\{\beta \in \Gamma:|\rho(\beta(x), z)-u|<\varepsilon\}, N=N_{\rho}(x, \varepsilon)$ and

$$
\Gamma(\beta)=\left\{\gamma \in \Gamma: \int_{0}^{t} \int_{0}^{2 \pi} \int_{u}^{t} 1_{\Phi_{z}^{-1} \beta N}\left(\tanh u e^{i \theta}\right) 1_{\Phi_{z}^{-1} \gamma N}\left(\tanh v e^{i \theta}\right) d v d \theta d u>0\right\}
$$

Denote by $\Lambda_{\gamma}$ the angle (set) subtended by $\Phi_{z}^{-1} \gamma N$ at 0 . Clearly

$$
\Gamma(\beta)=\left\{\gamma \in \Gamma: \rho\left(0, \Phi_{z}^{-1} \gamma(x)\right) \geq \rho\left(0, \Phi_{z}^{-1} \beta(x)\right)-\varepsilon, \Lambda_{\gamma} \cap \Lambda_{\beta} \neq \varnothing\right\} .
$$

Now one can calculate that for $y \in H, \rho(0, y)>\varepsilon$, the angle subtended by $N_{\rho}(y, \varepsilon)$ at 0 is

$$
\left\{\theta \in[0,2 \pi):\|\theta-\arg y\|<\sin ^{-1} \frac{\left(1-|y|^{2}\right) \tanh \varepsilon}{|y|\left(1-\tanh ^{2} \varepsilon\right)}\right\}
$$

where $y=|y| e^{i \arg y}, 0 \leq \arg y<2 \pi$ and $\|\theta-\varphi\|=\min \{|\theta-\varphi|,(2 \pi-|\theta-\varphi|)\}$. This shows that if $\rho\left(0, \Phi_{z}^{-1} \beta(x)\right) \leq \rho\left(0, \Phi_{z}^{-1} \gamma(x)\right)+\varepsilon$ and $\Lambda_{\beta} \cap \Lambda_{\gamma} \neq \varnothing$ then:

$$
\left\|\arg \Phi_{z}^{-1} \beta(x)-\arg \Phi_{z}^{-1} \gamma(x)\right\| \leq M e^{-2 \rho\left(0, \Phi_{z}^{-1} \beta(x)\right)}
$$

where $M$ is constant. Set

$$
\begin{aligned}
\Gamma_{1}(\beta)=\{\gamma \in \Gamma: & \rho\left(0, \Phi_{z}^{-1} \beta(x) \leq \rho\left(0, \Phi_{z}^{-1} \gamma(x)\right)+\varepsilon \quad\right. \text { and } \\
& \left.\left\|\arg \Phi_{z}^{-1} \beta(x)-\arg \Phi_{z}^{-1} \gamma(x)\right\| \leq M e^{-2 \rho\left(0, \Phi_{z}^{-1} \beta(x)\right)}\right\}
\end{aligned}
$$

Since $\Gamma(\beta) \subseteq \Gamma_{1}(\beta)$, we have that

$$
\psi(t, z) \leq \int_{0}^{t} \sum_{\beta \in \Gamma_{u}} \sum_{\gamma \in \Gamma_{1}(\beta)} \int_{0}^{2 \pi} \int_{u}^{t} 1_{\Phi_{2}^{-1} \gamma N}\left(\tanh v e^{i \theta}\right) d v d \theta .
$$

Fix $u, \beta \in \Gamma_{u}$ and $\gamma \in \Gamma_{1}(\beta)$. Then:

$$
\begin{aligned}
\int_{0}^{2 \pi} & \int_{u}^{t} 1_{\Phi \bar{z}^{-1} \gamma N_{\rho}(x, \varepsilon)}\left(\tanh v e^{i \theta}\right) d v d \theta \\
& =\int_{0}^{2 \pi} \int_{\tanh u}^{\tanh t} 1_{N_{\rho}\left(\Phi_{z}^{-1} \gamma(x), \varepsilon\right)}\left(r e^{i \theta}\right) \frac{d r d \theta}{1-r^{2}} \\
& =\int_{N_{\rho}(0, t) \backslash N_{\rho}(0, u)} \frac{1-|w|^{2}}{|w|} 1_{N_{\rho}\left(\Phi_{z}^{-1} \gamma(x), \varepsilon\right)}(w) d A(w) \\
& \leq\left(1-\tanh ^{2}\left(\rho\left(0, \Phi_{z}^{-1} \gamma(x)\right)-\varepsilon\right)\right) A_{1}\left(N_{\rho}\left(\Phi_{z}^{-1} \gamma(x), \varepsilon\right) \cap N_{\rho}(0, t) \backslash N_{\rho}(0, u)\right)
\end{aligned}
$$


where $d A_{1}(w)=(1 /|w|) d A(w)$. Hence, for $u$ and $\beta \in \Gamma_{u}$ fixed:

$$
\begin{aligned}
& \sum_{\gamma \in \Gamma_{1}(\beta)} \int_{0}^{2 \pi} \int_{u}^{t} 1_{\Phi_{z}^{-1} \gamma N}\left(\tanh v e^{i \theta}\right) d v d \theta \\
& \leq \sum_{\substack{\gamma \in \Gamma_{1}(\beta) \\
\rho\left(0, \Phi_{z}^{-1} \gamma(x) \leq t+\varepsilon\right.}}\left(1-\tanh ^{2}\left(\rho\left(0, \Phi_{z}^{-1} \gamma(x)\right)-\varepsilon\right) A_{1}\left(N_{\rho}\left(\Phi_{z}^{-1} \gamma(x), \varepsilon\right)\right)\right. \\
& \leq M^{\prime} \sum_{\substack{\gamma \in \Gamma_{1}(\beta) \\
\rho\left(0, \Phi_{z}^{-1} \gamma(x)\right) \leq t+\varepsilon}} e^{-2 \rho\left(0, \Phi_{z}^{-1} \gamma(x)\right)},
\end{aligned}
$$

since $A_{1}\left(N_{\rho}(0, \varepsilon)\right)<\infty$ and $A_{1}\left(N_{\rho}(x, \varepsilon)\right) \rightarrow A\left(N_{\rho}(0, \varepsilon)\right)$ as $|x| \rightarrow 1$. In order to proceed, we need further information about $\Gamma_{1}(\beta)$. Suppose that $u, V \in H$ satisfy $\rho(0, u) \leq \rho(0, V)$ and $|\arg u-\arg V| \leq K e^{-2 \rho(0, u)}$. Let $u^{1}=(|u| /|V|) V$. Then, since $0, u^{1}, V$ lie on a single geodesic:

$$
\rho(0, V)=\rho\left(0, u^{1}\right)+\rho\left(u^{1}, V\right) .
$$

Also, since $|u|=\left|u^{1}\right|, \rho(0, u)=\rho\left(0, u^{1}\right)$, and, by the triangle inequality:

$$
\rho\left(u^{1}, V\right) \geq \rho(u, V)-\rho\left(u, u^{1}\right) \text {. }
$$

Thus:

$$
\rho(0, V) \geq \rho(0, u)+\rho(u, V)-\rho\left(u, u^{1}\right)
$$

To estimate $\rho\left(u, u^{1}\right)$, we integrate $d s$ along the circular arc centred at 0 and joining $u$ to $u^{1}$, and obtain

$$
\rho\left(u, u^{l}\right) \leq \frac{|u|}{1-|u|^{2}} K e^{-2 \rho(0, u)} \leq \frac{K}{2}
$$

Hence, $\rho(0, V) \geq \rho(0, u)+\rho(u, V)-(K / 2)$. If, instead of $\rho(0, u) \leq \rho(0, V)$, we only suppose that $\rho(0, u) \leq \rho(0, V)+\varepsilon$, then we obtain

$$
\rho(0, V) \geq \rho(0, u)+\rho(u, V)-\max \{K / 2,2 \varepsilon\} .
$$

Now set $\Gamma_{2}(\beta)=\beta^{-1} \Gamma_{1}(\beta)$. If $\gamma \in \Gamma_{2}(\beta)$, then $\beta \gamma \in \Gamma_{1}(\beta)$ and so

$$
\begin{gathered}
\rho\left(0, \Phi_{z}^{-1} \beta(x)\right) \leq \rho\left(0, \Phi_{z}^{-1} \beta \gamma(x)\right)+\varepsilon \\
\left\|\arg \Phi_{z}^{-1} \beta(x)-\arg \Phi_{z}^{-1} \beta \gamma(x)\right\| \leq M e^{-2 \rho\left(0, \Phi_{z}^{-1} \beta(x)\right)}
\end{gathered}
$$

Thus, from the above,

$$
\begin{aligned}
\rho\left(0, \Phi_{z}^{-1} \beta \gamma(x)\right) & \geq \rho\left(0, \Phi_{z}^{-1} \beta(x)\right)+\rho\left(\Phi_{z}^{-1} \beta(x), \Phi_{z}^{-1} \beta \gamma(x)\right)-\max \{M / 2,2 \varepsilon\} \\
& =\rho(y, \beta(x))+\rho(x, \gamma(x))-\max \{M / 2,2 \varepsilon\}-\varepsilon,
\end{aligned}
$$

(since $\rho(y, z)<\varepsilon$ ), which shows that

$$
\sum_{\substack{\gamma \in \Gamma_{1}(\beta) \\ \rho\left(0, \Phi_{z}^{-1} \gamma(x)\right) \leq t+\varepsilon}} e^{-2 \rho\left(0, \Phi_{z}^{-1} \gamma(x)\right)} \leq M^{\prime \prime} e^{-2 \rho(y, \beta(x))} \sum_{\substack{\gamma \in \Gamma_{2}(\beta) \\ \rho\left(0, \Phi_{z}^{-1} \beta \gamma(x)\right) \leq t+\varepsilon}} e^{-2 \rho(x, \gamma(x))} .
$$

Now, $\gamma \in \Gamma_{2}(\beta), \rho\left(0, \Phi_{z}^{-1} \beta \gamma(x)\right) \leq t+\varepsilon$ implies that

$$
\begin{aligned}
\rho(x, \gamma(x)) & \leq \rho\left(0, \Phi_{z}^{-1} \beta \gamma(x)\right)-\rho(y, \beta(x))+\max \{M / 2,2 \varepsilon\}+\varepsilon \\
& \leq t+\max \{M / 2,2 \varepsilon\}+2 \varepsilon .
\end{aligned}
$$


Thus:

$$
\sum_{\substack{\gamma \in \Gamma_{1}(\beta) \\ \rho\left(0, \Phi_{z}^{-1} \gamma(x) \leq t+\varepsilon\right.}} e^{-2 \rho\left(0, \Phi_{z}^{-1} \gamma(x)\right)} \leq M^{\prime \prime} e^{-2 \rho(y, \beta(x))} a_{\Gamma}(x, x ; t+\max \{M / 2,2 \varepsilon\}+2 \varepsilon)
$$

and therefore:

$$
\psi(t, z) \leq M^{\prime \prime} a_{\Gamma}(x, x ; t+\max \{M / 2,2 \varepsilon\}+2 \varepsilon) \int_{0}^{t} \sum_{\beta \in \Gamma_{u}} e^{-2 \rho(y, \beta(x))} d u
$$

To finish, we note that:

$$
\begin{gathered}
\int_{0}^{t} \sum_{\beta \in \Gamma_{u}} e^{-2 \rho(y, \beta(x))} d u \leq 4 \varepsilon a_{\Gamma}(y, x ; t+2 \varepsilon) \\
a_{\Gamma}(u, V: t) \leq e^{2 \rho\left(u, u^{1}\right)+2 \rho\left(V, V^{1}\right)} a_{\Gamma}\left(u^{1}, V^{1} ; t+\rho\left(u, u^{1}\right)+\rho\left(V, V^{1}\right)\right) \\
\varlimsup_{t \rightarrow \infty} \frac{a_{\Gamma}(x, x, t+M)}{a_{\Gamma}(x, x, t)}<\infty
\end{gathered}
$$

obtaining:

$$
\begin{aligned}
\int_{\Delta_{y}} S_{t}\left(1_{\Delta_{x}}\right)^{2} d m_{\Gamma} & =\int_{N_{\rho}(y ; \varepsilon)} \psi(t, z) d A(z) \\
& \leq M^{\prime \prime \prime} a_{\Gamma}(y, x, t)^{2} \\
& \leq M^{\prime \prime \prime \prime}\left(\int_{\Delta_{v}} S_{t}\left(1_{\Delta_{x}}\right) d m_{\Gamma}\right)^{2}
\end{aligned}
$$

by proposition 1 .

Proposition 1 shows that a hyperbolic surface with recurrent geodesics is necessarily of divergence type. The fact that a divergence type surface has some recurrent geodesics will follow from lemma 1 and the next lemma (which is a continuous time version of the Borel-Cantelli lemma of Renyi mentioned in the introduction).

LemMa 2. Suppose that $(X, \mathscr{B}, \mu)$ is a finite measure space and that for $t>0, A_{t} \in B$ so that $1_{A_{t}}(x)$ is a jointly measurable function of $(x, t)$. If $\int_{0}^{\infty} \mu\left(A_{\imath}\right) d t=\infty$ and

$$
\varliminf_{t \rightarrow \infty} \int_{0}^{t} \int_{0}^{t} \mu\left(A_{u} \cap A_{V}\right) d u d V /\left(\int_{0}^{t} \mu\left(A_{s}\right) d s\right)^{2}=K<\infty
$$

then

$$
\mu\left(\left\{x \in X: \int_{0}^{\infty} 1_{A_{t}}(x) d t=\infty\right\}\right) \geq 1 / K .
$$

Proof. Set $b(t)=\int_{0}^{t} \mu\left(A_{s}\right) d s, \Phi_{t}=(1 / b(t)) \int_{0}^{t} 1_{A_{s}} d s$, and $A_{\infty}=\left\{x \in X: \int_{0}^{\infty} 1_{A_{s}}(x) d s=\right.$ $\infty\}$. Since $b(t) \rightarrow \infty$ we have that $\Phi_{t}(x) \rightarrow_{t \rightarrow \infty} 0$ for every $x \in A_{\infty}^{c}$. Also, there exists $t_{n} \rightarrow \infty$ so that

$$
\int_{X} \Phi_{t_{n}}^{2} d \mu=K_{n} \underset{n \rightarrow \infty}{\longrightarrow} K
$$


This means that the sequence $\left\{\Phi_{t_{n}}\right\}$ is uniformly integrable on $X$ (and hence on $\left.A_{\infty}^{c}\right)$. Therefore

$$
\int_{A_{\infty<}^{c}} \Phi_{t_{n}} d \mu \underset{n \rightarrow \infty}{\longrightarrow} 0
$$

(because $\Phi_{t_{n}} \rightarrow 0$ on $A_{\infty}^{c}$ ). But, $\int_{X} \Phi, d \mu=1$ for every $t$, so

$$
\int_{A_{\infty}} \Phi_{t_{n}} d \mu \underset{n \rightarrow \infty}{\longrightarrow} 1 \text {. }
$$

By the Cauchy-Schwartz inequality:

$$
\int_{A_{\infty}} \Phi_{n_{n}} d \mu \leq \sqrt{\mu\left(A_{\infty}\right)} \sqrt{K_{n}}
$$

and so

$$
\mu\left(A_{\infty}\right) K=\varliminf_{n \rightarrow \infty} \mu\left(A_{\infty}\right) K_{n} \geq 1
$$

Thus, we obtain that if $\Delta_{x}^{\Gamma} \subseteq X_{\Gamma}$ where $\Gamma$ is divergence type, then

$$
m_{\Gamma}\left(\left\{\omega \in X_{\Gamma}: \int_{0}^{t} 1_{\Delta_{x}^{\Gamma}} \cdot \varphi_{\Gamma}^{t}(\omega) d t=\infty\right\}\right)>0,
$$

(i.e. there are some recurrent geodesics), and hence, by [8]:

the geodesic flow is conservative and ergodic.

This completes the proof of the advertised part of theorem 1 .

Since we now know that the geodesic flow on a divergence type surface is conservative and ergodic, the inequality (5) tells us that it is rationally ergodic. The next proposition completes the proof of theorem 2 by identifying the asymptotic type of the flow.

Proposition 2. Let $\Gamma$ be of divergence type. Then there exists $a_{\Gamma}(t)$ such that

$$
\frac{S_{t}(f)}{a_{\Gamma}(t)} \leadsto \int_{X_{\Gamma}} f d m_{\Gamma}
$$

for every $f \in L^{1}\left(m_{\Gamma}\right)$, where $S_{i}(f)=\int_{0}^{t} f \cdot \varphi_{\Gamma}^{s} d s$. Moreover, for every $x, y \in H$ :

$$
a_{\Gamma}(x, y ; t) \sim a_{\Gamma}(t) / 4 \pi^{2} \quad \text { as } t \rightarrow \infty .
$$

Proof. Clearly, any such constants $a(t)$ must be defined uniquely up to asymptotic equality. Thus, to prove the proposition, it suffices to show that for any $x, y \in H$,

$$
\frac{S_{t}(f)}{a_{\Gamma}(x, y ; t)} \leadsto \int_{X_{\Gamma}} f d m_{\Gamma} / 4 \pi^{2} \quad \text { for } f \in L^{1}\left(m_{\Gamma}\right) \text {. }
$$

Choose $x, y \in H$. Evidently $a_{\Gamma}(x, y ; t)=a_{\Gamma}(x, \gamma(y) ; t)$ for $\gamma \in \Gamma, t>0$. Thus, without losing generality, we may assume that $\rho(x, y) \leq \rho(x, \gamma(y))$ for every $\gamma \in \Gamma, \gamma \neq e$. From this, we deduce that there is a fundamental domain $\mathscr{F}$ for $\Gamma$ with $x, y \in \mathscr{F}^{0}$. Hence, for $\varepsilon>0$ small enough, proposition 1 and lemma 1 apply to 
$\Delta_{x}^{\varepsilon}=N_{\rho}(x, \varepsilon) \times[0,2 \pi)$ and $\Delta_{y}^{\varepsilon}=N_{\rho}(y ; \varepsilon) \times[0,2 \pi)$. To begin, fix $\varepsilon>0$ small enough and set

$$
a(t)\left(=a^{\varepsilon}(t)\right)=\int_{\Delta_{x}^{\varepsilon}} S_{t}\left(1_{\Delta_{r}^{\varepsilon}}\right) d m_{\Gamma} / m_{\Gamma}\left(\Delta^{\varepsilon}\right)^{2}
$$

Then, clearly:

$$
\int_{\Delta_{x}^{e}}\left(S_{t}\left(1_{\Delta_{y}^{e}}\right) / a(t)\right) d m_{\Gamma}=m_{\Gamma}\left(\Delta^{\varepsilon}\right)^{2}
$$

and, by lemma 1:

$$
\sup _{t>1} \int_{\Delta_{x}^{*}}\left(S_{t}\left(1_{\Delta_{y}^{t}}\right) / a(t)\right)^{2} d m_{\Gamma}<\infty
$$

We show that $S_{t}(f) / a(t) \leadsto \int_{X_{\Gamma}} f d m_{\Gamma}$. To do this, suppose $t_{k} \rightarrow \infty$. There exists a subsequence (also denoted by $t_{k}$ ) and $\Phi \in L^{2}\left(\Delta_{x}^{\varepsilon}\right)$ so that

$$
S_{t_{k}}\left(1_{\Delta_{y}^{c}}^{c}\right) / a\left(t_{k}\right) \rightarrow \Phi \quad \text { weakly in } L^{2}\left(\Delta_{x}^{\varepsilon}\right) \text {. }
$$

Clearly $\int_{\Delta_{y}^{\varepsilon}} \Phi d m_{\Gamma}=m_{\Gamma}\left(\Delta^{\varepsilon}\right)^{2}$. There exists a further subsequence (still denoted by $t_{n}$ ) satisfying:

$$
\left|\int_{\Delta_{x}^{\varepsilon}}\left(\left(S_{t_{m}} / a\left(t_{m}\right)\right)-\Phi\right)\left(\left(S_{t_{n}} / a\left(t_{n}\right)\right)-\Phi\right) d m_{\Gamma}\right|<1 / 2^{n},
$$

for $1 \leq m \leq n-1$. In this situation:

$$
\frac{1}{N} \sum_{n=1}^{N}\left(S_{t_{n}}\left(1_{\Delta_{y}^{\varepsilon}}^{\varepsilon}\right) / a\left(t_{n}\right)\right) \rightarrow \Phi \quad \text { a.e. on } \Delta_{x}^{\varepsilon} .
$$

Now the set on which this convergence takes place is clearly $\varphi_{\Gamma}^{s}$-invariant, as is the limit function $\Phi$.

By ergodicity of $\varphi_{\Gamma}$, the convergence set (containing $\Delta_{x}^{\varepsilon}$ ) must be almost all of $X_{\Gamma}$, and the limit function $\Phi$ must be constant. But $\int_{\Delta_{x}^{e}} \Phi d m_{\Gamma}=m_{\Gamma}\left(\Delta^{\varepsilon}\right)^{2}$, so $\Phi=m_{\Gamma}\left(\Delta_{y}^{\varepsilon}\right)$ and

$$
\frac{1}{N} \sum_{n=1}^{N}\left(S_{t_{n}}\left(1_{\Delta_{r}}^{\varepsilon}\right) / a\left(t_{n}\right)\right) \rightarrow m_{\Gamma}\left(\Delta^{\varepsilon}\right) \text { a.e. }
$$

and, by Hopf's ratio theorem:

$$
\frac{1}{N} \sum_{n=1}^{N}\left(S_{t_{n}}(f) / a\left(t_{n}\right)\right) \rightarrow \int_{X_{\Gamma}} f d m_{\Gamma} \quad \text { a.e. } \quad \text { for all } f \in L^{1}
$$

This proves that $S_{t}(f) / a(t) \leadsto \int_{X} f d m_{\Gamma} \quad$ for all $f \in L^{1}$.

The above is true for $a(t)=a^{\varepsilon}(t)$ for $\varepsilon>0$ small enough. Thus, fixing $\varepsilon_{0}$ small enough, we have that $a^{\varepsilon}(t) \sim a^{\varepsilon_{0}}(t)$ as $t \rightarrow \infty$ for every $0<\varepsilon<\varepsilon_{0}$. But, by proposition 1 , we have that

$$
\varlimsup_{t \rightarrow \infty} \frac{a^{\varepsilon}(t)}{a_{\Gamma}(x, y ; t)} \leq e^{4 \varepsilon} / 4 \pi^{2}, \quad \varliminf_{t \rightarrow \infty} \frac{a^{\varepsilon}(t)}{a_{\Gamma}(x, y ; t)} \geq e^{-4 \varepsilon} / 4 \pi^{2} .
$$


Thus for $\varepsilon>0$ small enough:

$$
a^{\varepsilon}(t) \sim \frac{a_{\Gamma}(x, y ; t)}{4 \pi^{2}} \quad \text { as } t \rightarrow \infty
$$

If $m_{\Gamma}\left(X_{\Gamma}\right)<\infty$ then, by the Birkhoff ergodic theorem, $a_{\Gamma}(t) \sim t / m_{\Gamma}\left(X_{\Gamma}\right)$ and so, by proposition 2 , we recover the well known result that

$$
a(x, y ; t) \sim \frac{4 \pi^{2} t}{m_{\Gamma}\left(X_{\Gamma}\right)}=\frac{2 \pi t}{A\left(H_{\Gamma}\right)} \quad \text { as } t \rightarrow \infty .
$$

In this case $\left(m_{\Gamma}\left(X_{\Gamma}\right)<\infty\right)$ we can do better. Hedlund [5], proved that the geodesic flow is mixing, i.e.

$$
m_{\Gamma}\left(A \cap \varphi_{\Gamma}^{-t} B\right) \underset{t \rightarrow \infty}{\longrightarrow} m_{\Gamma}(A) m_{\Gamma}(B) / m_{\Gamma}\left(X_{\Gamma}\right)
$$

In particular:

$$
\int_{t-c}^{t+c} m_{\Gamma}\left(\Delta_{x}^{\varepsilon} \cap \varphi_{\Gamma}^{-s} \Delta_{y}^{\varepsilon}\right) d s \underset{t \rightarrow \infty}{\longrightarrow} 2 c m_{\Gamma}\left(\Delta_{x}^{\varepsilon}\right) m_{\Gamma}\left(\Delta_{y}^{\varepsilon}\right) / m_{\Gamma}\left(X_{\Gamma}\right)
$$

for $c, \varepsilon>0$.

On the other hand, a calculation along the lines of proposition 1 shows that

$$
\begin{aligned}
\alpha_{0}(t) e^{-4 \varepsilon} A(N)^{2} u(x, y, c-2 \varepsilon, t) & \leq \int_{t-c}^{t+c} m_{\Gamma}\left(\Delta_{x}^{\varepsilon} \cap \varphi_{\Gamma}^{-s} \Delta_{y}^{\varepsilon}\right) d s \\
& \leq e^{4 \varepsilon} \alpha_{1}(t) u(x, y, c+2 \varepsilon, t) A(N)^{2},
\end{aligned}
$$

where $u(x, y, c, t)=\sum_{y \in \Gamma,|\rho(x, y(y))-t| \leq c} e^{-2 \rho(x, y(y))}$ and $\alpha_{0}(t), \alpha_{1}(t) \rightarrow_{t \rightarrow \infty} 1$, from which we obtain that

$$
u(x, y, c, t) \underset{t \rightarrow \infty}{\longrightarrow} \frac{4 \pi c}{A\left(H_{\Gamma}\right)} \quad \text { for } c>0 .
$$

From this one can obtain that

$$
e^{-2 t} \#\{\gamma \in \Gamma: \rho(x, \gamma(y)) \leq t\} \underset{t \rightarrow \infty}{\longrightarrow} \pi / A\left(H_{\Gamma}\right) ;
$$

(see Patterson [11] where this, and refinements thereof, are obtained by different methods).

\section{REFERENCES}

[1] J. Aaronson. On the ergodic theory of non-integrable functions and infinite measure spaces. Israel J. Math. 27 (1977), 163-173.

[2] J. Aaronson. Rational ergodicity and a metric invariant for Markov shifts. Israel J. Math. 27 (1977), 93-123.

[3] J. Aaronson. On the pointwise ergodic behaviour of transformations preserving infinite measures. Israel J. Math. 32 (1979), 67-82.

[4] L. R. Ford. Automorphic Functions. McGraw-Hill, 1929 and Chelsea, 1951.

[5] G. Hedlund. The dynamics of geodesic flows. Bull. Amer. Math. Soc. 45 (1939), 241-260.

[6] E. Hopf. Fuchsian groups and ergodic theory. Trans. Amer. Math. Soc. 39 (1936), 299-314.

[7] E. Hopf. Ergodentheorie. Chelsea, (1948). 
[8] E. Hopf. Statistik der geodätischen Linien in Mannigfaltigkeiten negativer Krümmung. Ber. Verh. Sächs. Akad. Wiss. Leipzig 91 (1939), 261-304.

[9] E. Hopf. Ergodic theory and the geodesic flow. Bull. Amer. Math. Soc. 77 (1972), 863-871.

[10] P. Nicholls. Transitivity properties of Fuchsian groups. Canad. J. Math. 28 (1976), 805-814.

[11] S. J. Patterson. A lattice-point problem in hyperbolic space. Mathematika 22 (1975), 81-88.

[12] H. Poincaré. Oeuvres. Gauthier-Villars: Paris, 1956.

[13] M. Rees. Checking ergodicity of some geodesic flows with infinite Gibbs measure. Ergod. Th. \& Dynam. Sys. 1 (1981), 107-133.

[14] A. Renyi. Probability Theory. North Holland, (1970). (p. 391).

[15] D. Sullivan. On the ergodic theory at infinity of an arbitrary discrete group of hyperbolic motions. Annals of Math. Studies, 97 (1979), 465-496.

[16] D. Sullivan. The density at infinity of a discrete group of hyperbolic motions. Publ. Math. IHES S0 (1980), 171-202.

[17] W. Thurston. Geometry and topology of 3-manifolds. Preprint, (1978).

[18] M. Tsuji. Potential theory in modern function theory. Maruzen Co. Ltd: Tokyo, 1959. 